\title{
La biblioteca híbrida como oportunidad para situar a la biblioteca pública en el centro de la sociedad
}

\author{
Por Agnès Felis Prósper
}

\begin{abstract}
Resumen: La introducción de las nuevas tecnologías en el ámbito bibliotecario ha iniciado un proceso de digitalización y transición hacia el autoservicio parcial en las bibliotecas que plantea nuevos retos y nuevas oportunidades para los profesionales. La rentabilidad económica, el ahorro de tiempo y la dedicación a tareas menos rutinarias son algunas de las ventajas que conlleva. En el marco del II Seminario de gestión de bibliotecas públicas, organizado por la Fundación Bertelsmann en Barcelona, algunos expertos europeos abordaron la cuestión planteándola como oportunidad para devolverle a la biblioteca un papel referencial en la sociedad.
\end{abstract}

Palabras clave: Biblioteca híbrida, Nuevas tecnologías de la información y la comunicación, Autoservicio, Recursos electrónicos, Digitalización.

\section{Title: The hybrid library as an opportunity for positioning the public library at society's centre}

\begin{abstract}
The introduction of information and communication technologies in librarian circles has led to a process of digitisation and of transition to partial self-service in libraries, giving rise to new challenges and opportunities for professionals. Profitability, timesavings, and dedication to less monotonous tasks are some of the benefits it entails. During the 2nd Public Libraries Management Seminar, organised by the Fundación Bertelsmann in Barcelona, several European experts addressed this issue which they consider to represent an opportunity for libraries to become a reference point for society.
\end{abstract}

Keywords: Hybrid library, ICT, Information and communication technologies, Self-service, Electronic resources, Digitisation.

LAS TRANSFORMACIONES que están teniendo lugar en prácticamente todos los ámbitos de nuestra sociedad a medida que se extienden las nuevas tecnologías de la información y la comunicación (TIC) alcanzan tal magnitud en el caso de las bibliotecas que en los noventa se acuñó un término para bautizar al nuevo concepto de biblioteca resultante de su aplicación. En un primer momento, se utilizó la denominación 'biblioteca híbrida' para hacer referencia a un espacio en el que ya no sólo se ofrecen unos fondos documentales en papel, sino en el que también se utilizan como fuentes de informa- ción recursos electrónicos. De este tema, entre otros, se trató en el II Seminario de gestión de bibliotecas públicas, organizado por la Fundación Bertelsmann en Barcelona.

\section{Las dos vertientes de la biblioteca híbrida}

Esta definición, según la cual lo que la caracteriza es la combinación de fuentes tradicionales y digitales no contempla, sin embargo, un fenómeno que hoy se está dando ya en algunos países de forma simultánea. Algo que, si bien no anula ni invalida la primera concepción, sí añade una nueva dimensión basada en la forma, o más bien en el lugar, desde el cual se accede a dicha información. Esta segunda aproximación al término 'híbrida' incluiría el hecho de que los servicios que se ofrecen estén a disposición del usuario tanto de forma presencial (estando físicamente en la biblioteca) como remota, a través de internet o de redes de área local.

Esta dualidad en cuanto a la propia definición puede generar hoy día cierta confusión, especialmente en los medios y foros de debate internacionales en los que se utiliza una misma 'etiqueta' para hacer referencia a realidades muy distintas en términos del avance alcanzado en la implantación de las 


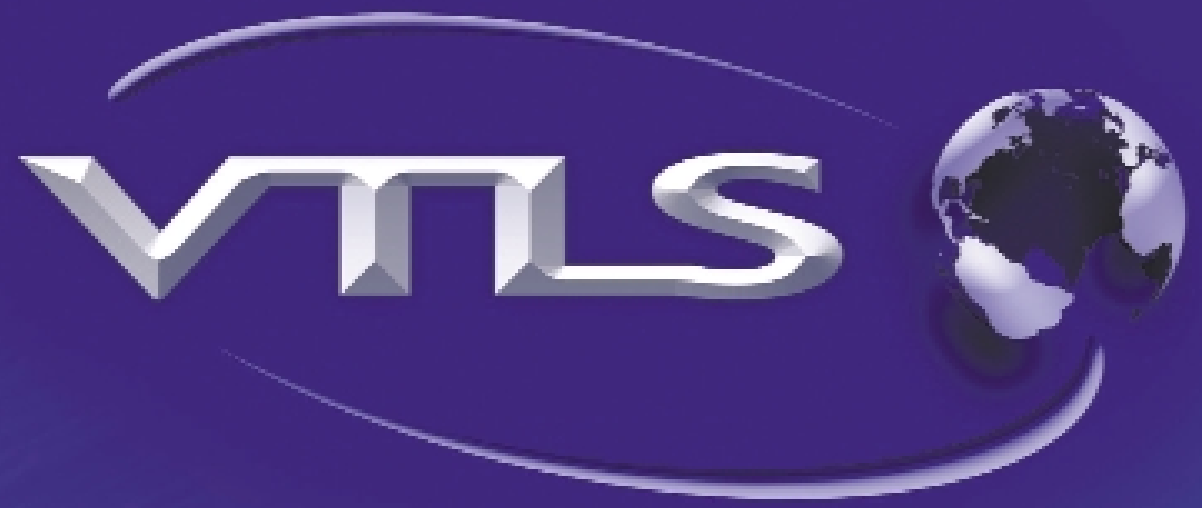

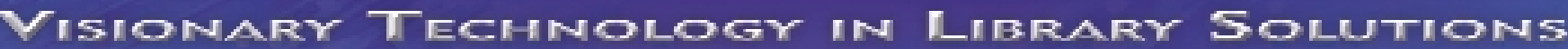

\section{Integramos avances tecnológicos para bibliotecas.}

\section{Hablamos tu idioma.}

Un sistema integrado de automatización con los últimos avances como

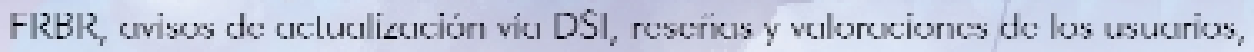
y una interfaz para dispositivos inteligentes (PDA).

Una solución de RFID de nuestra división VTRAX, que eombina nucstros años de experiencia bibliotecaria con la última tecnología en RFID. Procesar material nunca fue tan rápiclo como ahoro, con máquinos de autopréstamo para múltiples ejemplares, inventarios ágiles, conexiones sin cables y devoluciones de material que hacen el trabajo por ti. Además, FASTRAC funciono con cusulefuicr sisteme de culemulización.

Uno solución de tecnologin punto para bibliotecns digitales que permite crenr un sisteme de gestión de contenidos digitules en buse al software de código abierto Fedora'm. Ahora tu biblioteca puede utilizar este paquete de código abierto y obtener de VTLS formoción, soporte y funcianalidades añodidas.

Vortex es una potente herramienta que pone los registros de la biblioteca a dispessición de un número polenciculmorite ilimilado de usuarios. P'or cumplir con $\mathrm{OAl}$ y ser usuaria de Vortex, tu biblioteca será una referencia importante en cunnto a recursos comportidos y trabojo coordinado de colecciones. Además, ies de código abierto! 
nuevas tecnologías. A medida que ambos procesos avancen y confluyan, los criterios se unificarán de forma natural y llegará un momento en el que no hará falta utilizar el apelativo 'híbrida' porque todas las bibliotecas lo serán. De momento, sin embargo, limitaremos el alcance del término y utilizaremos el concepto en su primera acepción, que la entiende como aquella en la que el soporte impreso se combina con fuentes de información en formato digital.

Resulta emblemático, desde este punto de vista, uno de los datos que presentó la directora de la biblioteca pública de Estocolmo, Inga Lundén, durante el seminario, pues la mitad de las consultas que recibe actualmente la biblioteca (ubicada en un majestuoso edificio "de cuando se creía que todo el conocimiento podía estar contenido en un solo lugar") se pueden resolver con la colección propia, mientras que para el cincuenta por ciento restante hace falta recurrir a internet.

\section{La biblioteca como lugar de encuentro}

No obstante, esto no significa en absoluto que el rol que desempeña la biblioteca como espacio físico quede desvirtuado, ya que en las funciones que cumple como equipamiento público intervienen

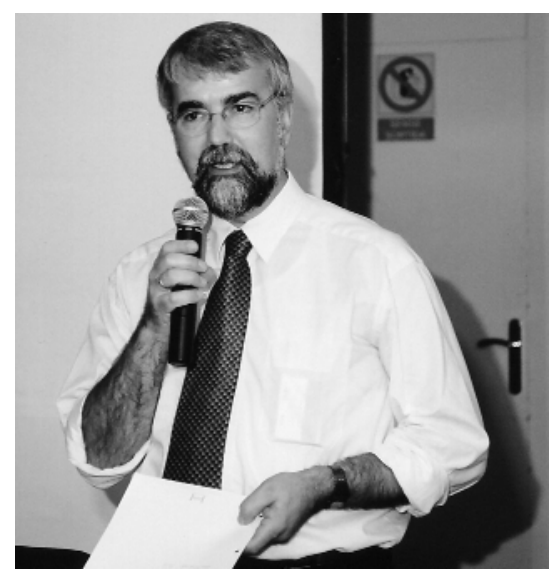

Ton van Vlimmeren

otros factores difíciles de reflejar en los informes estadísticos. La propia Lundén argumentó, por ejemplo, que en una sociedad en la que las viviendas son cada vez más pequeñas (a lo que se suma la frecuente necesidad de compartirlas) la biblioteca adquiere un valor añadido como "lugar especial en el que coincide mucha gente concentrada, leyendo o estudiando" y se convierte en "un placer en sí mismo como espacio".

Su potenciación como lugar de encuentro es uno de los frentes en los que hoy libramos batalla los profesionales y entidades que trabajamos para que recupere su papel referencial en la sociedad. Pero para ello es necesario que se tenga una visión clara de hacia dónde se quiere avanzar, ya que si no sabemos cuál es el modelo de biblioteca que queremos construir no podremos hacer partícipes de nues-

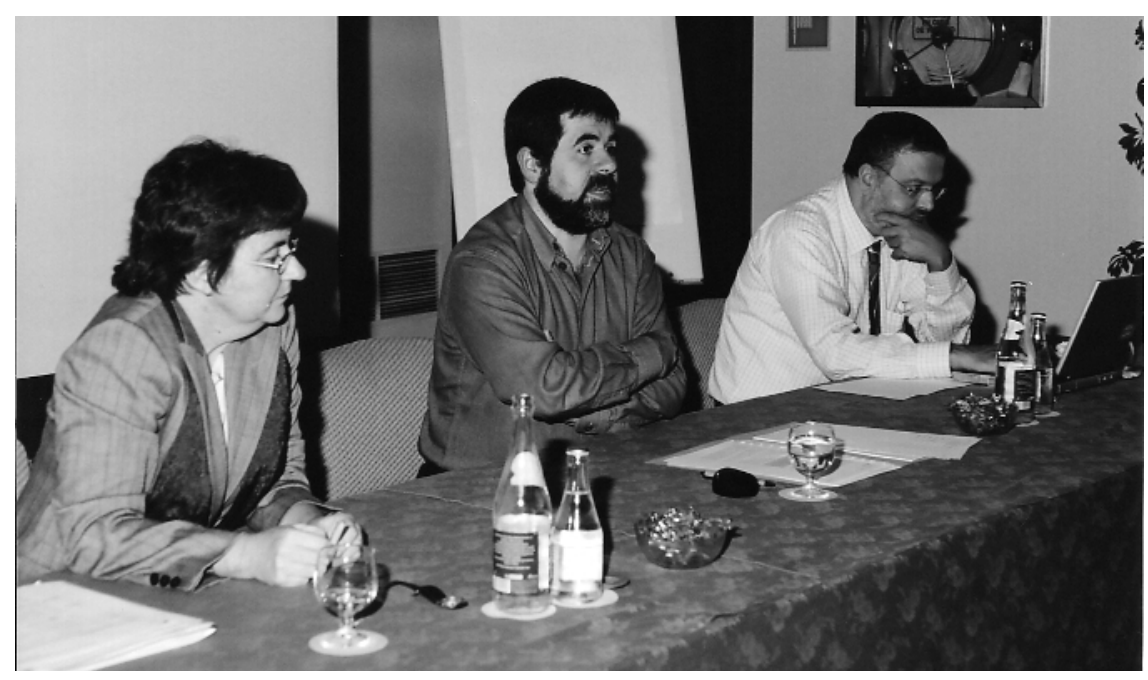

Eulàlia Espinàs, Jordi Sánchez y Manel Sanromà tras convicciones a los estamentos políticos. Y dicha implicación es crucial porque de su intervención depende que se disponga de los medios para alcanzar los objetivos.

Lundén subrayó la necesidad de que las bibliotecas se adelanten a los acontecimientos y estudien la forma de mantener e incrementar su peso específico dentro de la sociedad, puesto que incluso ahora, "un momento de gran éxito en el que la biblioteca está en la cresta de la ola, con más usuarios que los estadios deportivos, los museos o los teatros", se perciben síntomas de alejamiento entre ellas y la ciudadanía. Según una encuesta realizada en Suecia, todavía hay muchos usuarios potenciales que no aprovechan los servicios a pesar de que reúnen las condiciones objetivas para ser usuarios habituales: se trata de ciudadanos que leen y buscan información (normalmente en internet) pero que no identifican la biblioteca como un equipamiento pensado para ellos.

A pesar de que todos los encuestados estaban de acuerdo en calificar la biblioteca como algo "bueno y positivo" por definición, nadie parecía considerarse a sí mismo como usuario natural. Los jóvenes la veían como un lugar muy apropiado para la gente mayor, mientras que éstos consideraban que se trataba de un sitio ideal para aquellos. Y entre clases sociales sucedía algo similar: los encuestados con un alto poder adquisitivo opinaban que era un gran invento para la gente sin recursos, mientras que los ciudadanos de clase baja la percibían como algo demasiado intelectual para sus hábitos. La conclusión expresada por Lundén es irrebatible: "hay mucho trabajo por hacer en el terreno del marketing". Esta periodista y bibliotecaria manifestó su preocupación en términos dramáticos: "si la biblioteca pública no logra reafirmarse como primera opción en el ámbito de los 
servicios públicos de información, morirá junto con la última generación de políticos nostálgicos. Y eso significaría el fin del acceso libre y gratuito de la gente a la información".

\section{Gobierno electrónico: un espacio por conquistar}

Dadas las circunstancias, el replanteamiento de fondo que está suscitando el debate acerca de la biblioteca híbrida representa una gran oportunidad para las bibliotecas públicas, tal como constataron los asistentes al encuentro. Los 21 bibliotecarios de toda España que participaron en el seminario concluyeron, por ejemplo, que el vacío que existe actualmente en términos de oferta de servicios para implantar el gobierno electrónico en nuestro país supone un espacio que las bibliotecas públicas pueden conquistar. De este modo conseguirían atraer a los usuarios y situarse en el centro de la actividad pública ofreciendo un servicio con gran proyección, para el que además está dotada de forma natural por tratarse de una institución democrática con una función compensatoria que lima desigualdades y está gestionada por profesionales de la información capaces de generar contenidos, editarlos y facilitar su accesibilidad.

Los obstáculos, sin embargo, no son nada desdeñables. El liderazgo de la biblioteca como centro de información única topa, por ejemplo, con la atomización informativa de las distintas áreas de las administraciones públicas, cada una de las cuales establece canales propios de comunicación directa con los ciudadanos. A esta falta de coordinación se suma otro eslabón perdido que es el que debería vincular a las bibliotecas con los planes gubernamentales de desarrollo de las TIC, de los cuales han quedado excluidas. Así, mientras las bibliotecas centrales incorporan las nuevas tecnologías, los departamentos de nueva creación concebidos para promoverlas no las contemplan a la hora de diseñar sus programas y estrategias.

Parece lejano aún, en vista de la coyuntura, un proceso de sistematización similar al que se está llevando a cabo en otros países, entre los cuales destaca Singapur, donde el 99\% de la circulación de libros y documentos en esta ciudad-estado se efectúa mediante autoservicio. Según expuso en el marco del seminario el director de la Biblioteca Pública de Utrecht, Ton Van Vlimmeren, en el caso de los países escandinavos este porcentaje alcanza el $98 \%$ en las bibliotecas donde se trabaja con códigos de barras y cintas electromagnéticas, mientras que en el primer centro que implementó la tecnología por radiofrecuencia, un 92\% de la circulación la gestionan ya los propios usuarios.

\section{La reinvención del trabajo del bibliotecario}

Para Van Vlimmeren, la gran ventaja del autoservicio no está tanto en la comodidad de los usuarios (que les permite moverse más libremente) como en la oportunidad que conlleva para los bibliotecarios de reducir el tiempo que dedican a tareas rutinarias y poco cualificadas. El proceso de reinvención del trabajo del bibliotecario que se deriva de la automatización de ciertas operaciones (sobre todo los préstamos y las devoluciones) pasa por sacar un mayor partido a la formación de los profesionales, algunos de los cuales tan só-

\section{¿Recibes ya EPI en casa? \\ Por sólo 75 euros $+4 \%$ IVA puedes tener todos los meses tu copia particular y leerla cómodamente cuan- do quieras.}

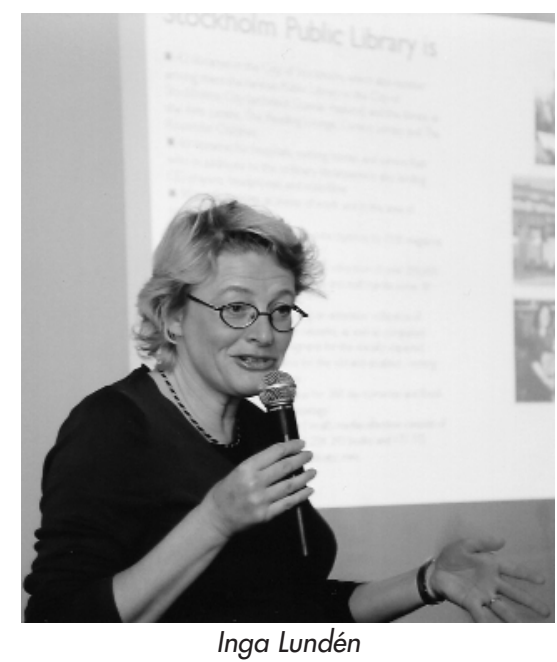

lo dedican 20 horas mensuales a tareas que requieren realmente los conocimientos que poseen.

Aun así, Van Vlimmeren reconoce que el personal que se siente reemplazado tiende a mostrarse renuente a este cambio, puesto que el miedo a perder el puesto de trabajo pesa más que el incentivo que supone la posibilidad de desempeñar tareas más cualificadas y mejor retribuidas. No obstante, este directivo no cree que dicho temor esté justificado porque "no hace falta despedir a nadie: unos se jubilan y otros deberán cubrir los puestos que se creen en las bibliotecas de nueva construcción, aparte de que a los que se queden hay que motivarlos y prepararlos para que desempeñen un papel más anfitrión, sabiendo qué tienen en la biblioteca y cómo atender mejor al usuario".

En cuanto a los costes iniciales que supone la implantación de este tipo de tecnologías, Van Vlimmeren avaló la rentabilidad del autoservicio con su experiencia, que ha demostrado que la inversión en estos sistemas se recupera en un periodo de entre cinco y seis años. No se puede decir lo mismo todavía de los robots que se están utilizando de forma experimental para tareas de clasificación en algunas bibliotecas (como por ejemplo la de Malmö, en Suecia) ya que la inversión que requieren es todavía demasiado alta y la viabilidad eco- 
nómica no está nada clara si los robots no son operados por colaboradores voluntarios.

Aun así, reconoció que en las bibliotecas muy pequeñas no tiene sentido, al menos de momento, introducir sistemas de autoservicio. Reivindicó, eso sí, la necesidad de desarrollar vías de cooperación para incrementar la fuerza de las bibliotecas en los foros de debate y en los ámbitos de negociación con los políticos. "Las bibliotecas tienden a ser olvidadas por los gobiernos porque no tienen una identidad clara y no saben exactamente qué rol tienen que desempeñar; no se adscriben netamente ni a la educación como centro de formación reglada, ni a la cultura como centros de actividades, ni a la información como lugar que ofrece servicios aparte de ser un espacio público. Pero el hecho de que los políticos no tengan una visión clara de cuál es la infraestructura necesaria para llevar a cabo la transformación que reclamamos debe ser aprovechado por los bibliotecarios para erigirse en líderes y asumir la iniciativa de presentarles soluciones." En este sentido puso como ejemplo a EUA, donde afirmó que las bibliotecas han sabido promocionarse para conseguir el dinero necesario.

\section{Perspectivas de futuro en España}

Tener modelos de referencia es sin duda partir con ventaja en el proceso de transformación hacia la biblioteca híbrida, tal como reconocieron los profesionales españoles tras una de las sesiones de trabajo que tuvieron lugar en el marco del encuentro. Nuestra realidad difiere mucho hoy día de la de Holanda, por ejemplo, donde existe un servicio central de bibliotecas, el NBD (Nederlandse Bibliotheek Dienst) que centraliza la demanda, la compra, el etiquetaje y la distribución de los libros. Esta entidad, creada por las bibliotecas hace 30 años sin ánimo de lucro, lee las novedades y asesora a las bibliotecas, a las que envía los ejemplares reencuadernados siguiendo un patrón de formato único. Pero evidentemente hubo un tiempo de adaptación y "un precio que hubo que pagar por ser los primeros".

En España la dispersión es mucho mayor y un nivel de coordinación similar al que representa el $N B D$ parece impensable, pero en cambio se dan circunstancias que pueden propiciar la recuperación de la biblioteca pública como centro de acceso a la información por parte de los ciudadanos. La baja cuota de penetración de internet en los hogares, por ejemplo, que hoy se sitúa entre un 40 y un 50 por ciento de la población (según datos aportados por Jordi Sánchez, director de la Fundació Jaume Bofill, en el marco del seminario) supone una oportunidad para posicionarse como proveedor y centro formador en nuevas tecnologías.

En todo caso, a lo largo del seminario se hizo patente la necesidad de aprovechar la situación actual y el proceso de transformación en que se halla inmersa la biblioteca pública para situarla en el centro de la sociedad. Hacerla más visible y potenciar su papel como lugar de encuentro y punto de referencia son dos de los ejes que marcarán las estrategias de futuro, que sin duda deberán contar con una realidad que, en palabras de Inga Lundén, se podría resumir diciendo que "vamos hacia una biblioteca híbrida en un mundo híbrido". La directora de la biblioteca de Estocolmo cerró su intervención apostando por la acción: "Aunque no veamos claro lo que tenemos delante, hay que avanzar". O, lo que es lo mismo: "caminante no hay camino, se hace camino al andar".

Agnès Felis Prósper, responsable de comunicación y publicaciones de la Fundación Bertelsmann. agnes.felis@fundacionbertelsmann.org 